\title{
Composite Adaptive Antidisturbance Control for Discrete-Time Switched System
}

\author{
Haibin Sun, ${ }^{1,2}$ Qiang Lu, ${ }^{1}$ and Linlin $\mathrm{Hou}^{3}$ \\ ${ }^{1}$ School of Automation, Hangzhou Dianzi University, Hangzhou, Zhejiang 310018, China \\ ${ }^{2}$ School of Engineering, Qufu Normal University, Rizhao, Shandong 276826, China \\ ${ }^{3}$ School of Information Science and Engineering, Qufu Normal University, Rizhao, Shandong 276826, China
}

Correspondence should be addressed to Haibin Sun; fengyun198212@163.com

Received 24 December 2015; Accepted 21 March 2016

Academic Editor: Juan R. Torregrosa

Copyright (C) 2016 Haibin Sun et al. This is an open access article distributed under the Creative Commons Attribution License, which permits unrestricted use, distribution, and reproduction in any medium, provided the original work is properly cited.

\begin{abstract}
A novel composite adaptive antidisturbance controller is developed for a class of discrete-time switched system. First, two composite adaptive observers are proposed to estimate the external disturbances and unknown parameters, respectively. Then, based on the estimation values, a composite adaptive antidisturbance controller is constructed, which can guarantee system has a good antidisturbance performance. A solvable sufficient condition is presented by using linear matrix inequalities (LMIs). Finally, a numerical example is shown to demonstrate the effectiveness of the proposed control approach.
\end{abstract}

\section{Introduction}

Disturbances widely exist in practical system and bring adverse effect of the control performance of the closedloop system. To enhance the antidisturbance ability of the considered system, many effective antidisturbance control schemes have been proposed, for example, nonlinear $H_{\infty}$ control $[1,2]$, nonlinear output regulation theory $[3,4]$, sliding mode control $[5,6]$, and disturbance observer based control (DOBC). DOBC technique has received more and more attention and has been applied to many kinds of control fields, such as hard disk drive systems $[7,8]$, robotic systems [9], grinding systems [10, 11], hypersonic vehicles $[12,13]$, spacecraft systems $[14,15]$, and general systems [1618]. In the DOBC scheme, an observer is constructed to estimate external disturbances and the estimation is used for feedforward compensation. Then a composite controller is obtained based on disturbance observer and conventional feedback control law, which can guarantee system has a good disturbance rejection performance and desired stability or tracking performance. When systems are subject to multiple disturbances, composite hierarchical antidisturbance control
(CHADC) methods have been given [19] via DOBC method and conventional antidisturbance control technique, for example, $H_{\infty}\left(L_{2}-L_{\infty}\right)$ control $[20,21]$, sliding mode control $[22,23]$, adaptive control [24, 25], and neural network [26]. The problem of robust stability analysis and robust controller design has been extensively investigated for discrete-time systems with uncertainty and/or disturbance. In [27, 28], two back-stepping controllers are proposed for the longitudinal dynamics of a generic hypersonic flight vehicle with neural networks based on discrete-time model. In [29], a composite antidisturbance controller has been developed for a class of discrete-time system with multiple disturbances. A composite hierarchical antidisturbance fault-tolerant controller has been developed for a class of discrete-time system with multiple disturbances and actuator fault via switching method in $[30]$. In $[29,30]$, they do not pay attention to estimating uncertain function and external disturbances for discretetime system at the same time. Hence, it is necessary to develop a novel antidisturbance controller for discrete-time system with multiple mismatched disturbances, that is, single harmonic or constant disturbances and another unexpected nonlinear signal presented as a nonlinear function. 
On the other hand, switched systems are a special class of hybrid system and can be used to describe many practical systems, for example, power electronics, embedded systems, chemical processes, computer-controlled systems, and automotive industries. Hence, the stability analysis and controller synthesis of switched system have received more and more attention during the last decade and many results have been developed [31-34]. By far, there are a number of methodologies on dealing with the stability analysis and control synthesis of switched systems, such as common Lyapunov function [31], multiple Lyapunov function [32], dwell time and average dwell time method [33], and switched Lyapunov function [34]. In [35], a CHADC method has been reported for a class of discrete-time switched system with multiple disturbances, but the disturbances are assumed to be described by an exogenous system or satisfy $\mathrm{H}_{2}$ norm.

In this paper, the problem of adaptive antidisturbance control for discrete-time switched system with disturbances is addressed. The disturbances are described by not only single harmonic or constant disturbances but also another unexpected nonlinear signal presented as a nonlinear function. In order to improve the disturbance rejection and robustness performance of systems, an adaptive antidisturbance controller is proposed via disturbance observer technique and adaptive control method. First, the composite adaptive observers are constructed to estimate disturbances with known partial information and unknown parameters. Then, an adaptive antidisturbance controller is developed based on estimation value and conventional feedback control law. Combining switched Lyapunov function method and linear matrix inequalities, a sufficient condition is presented to obtain the controller and observer gains. Finally, a numerical example is given to demonstrate the effectiveness of the proposed scheme.

\section{Problem Formulation and Preliminaries}

Consider the following discrete-time switched systems with time-varying delays described by

$$
\begin{aligned}
\Sigma_{0}: x(k+1)= & A_{\sigma(k)} x(k) \\
& +B_{\sigma(k)}\left(u(k)+d_{1}(k)+\phi^{T}(k) \theta(k)\right),
\end{aligned}
$$

where $x(k) \in \mathbb{R}^{n}$ is the states; $u(k)$ is the control input. $\phi(k)$ is a bounded function, which satisfies $\|\phi(k)\| \leq \phi$. Consider $\sigma(k): \mathbb{Z}^{+} \rightarrow \mathcal{N}=\{1,2, \ldots, N\}$ is the switching signal, which specifies which subsystem will be activated at a certain discrete-time instant and $A_{i}, B_{i}, i \in \mathcal{N}$, are constant matrices with appropriate dimensions. $d_{1}(k)$ is the external disturbances, which is described by Assumption 1 . $\theta(k)$ describes a time-varying uncertain parametric vector to be updated or estimated.

Assumption 1 (see [29]). The disturbances $d_{1}(k)$ are generated by the exogenous system

$$
\begin{gathered}
\omega(k+1)=W \omega(k), \\
d_{1}(k)=V \omega(k),
\end{gathered}
$$

where $\omega(k) \in \mathbb{R}^{m} \cdot W \in R^{m \times m}$ and $V \in R^{1 \times m}$ are known matrices.

Assumption 2. Unknown parametric vector $\theta$ in (1) can be supposed to satisfy the following formula:

$$
\theta(k+1)=\Gamma \theta(k)
$$

where $\Gamma \in R^{\kappa \times \kappa}$ is a proper matrix and the initial condition is unknown.

Remark 3. Assumption 2 is a rational assumption condition. Because some unknown parameters can be described by a given model in practical system, for example, in spacecraft system, the inertial matrix can be defined by the given model via choosing appropriate parameters.

Here we are devoted to designing a state feedback controller by the following formula:

$$
u(k)=K_{1 \sigma(k)} x(k)-\widehat{d}_{1}(k)-\phi^{T}(k) \hat{\theta}(k),
$$

where $\widehat{d}_{1}(k)$ and $\widehat{\theta}(k)$ are the estimation of the disturbances $d_{1}(k)$ and $\theta(k) \cdot \widehat{d}_{1}(k)$ is obtained by the following disturbance observer:

$$
\begin{aligned}
& \widehat{\omega}(k)=v(k)-L x(k), \\
& \nu(k+1)=W \widehat{\omega}(k)+L\left(A_{\sigma(k)} x(k)\right. \\
& \left.+B_{\sigma(k)}\left(u(k)+\widehat{d}_{1}(k)+\phi^{T}(k) \hat{\theta}(k)\right)\right), \\
& \widehat{d}_{1}(k)=V \widehat{\omega}(k) .
\end{aligned}
$$

$\theta(k)$ is estimated by the following adaptive law:

$$
\begin{aligned}
& \widehat{\theta}(k)=v_{0}(k)-L_{0} x(k), \\
& v_{0}(k+1)=\Gamma \widehat{\theta}(k)+L_{0}\left(A_{\sigma(k)} x(k)\right. \\
& \left.\quad+B_{\sigma(k)}\left(u(k)+\widehat{d}_{1}(k)+\phi^{T}(k) \hat{\theta}(k)\right)\right) .
\end{aligned}
$$

Remark 4. In this paper, we assume that the switching signal $\sigma(k)$ is not known a priori but its instantaneous value is available in real time [36]. Here we only consider the case of synchronous switching; that is, the controller switches just as the system $\Sigma_{0}$ does. yields

$$
\text { Defining } e_{\omega}(k)=\omega(k)-\widehat{\omega}(k) \text { and } e_{\theta}(k)=\theta(k)-\widehat{\theta}(k)
$$

$$
\begin{aligned}
& e_{\omega}(k+1)=(W+L V) e_{\omega}(k)+L \phi^{T}(k) e_{\theta}(k), \\
& e_{\theta}(k+1)=\left(\Gamma+L_{0} \phi^{T}(k)\right) e_{\theta}(k)+L_{0} B_{\sigma(k)} e_{\omega}(k) .
\end{aligned}
$$


Applying controller (4) to system $\Sigma_{0}$ and combining (2) and (6), we obtain

$$
\begin{aligned}
x(k+1)= & \left(A_{\sigma(k)}+B_{\sigma(k)} K_{\sigma(k)}\right) x(k) \\
& +B_{\sigma(k)}\left(d_{1}(k)-\widehat{d}_{1}(k)\right) \\
& +B_{\sigma(k)}\left(\phi^{T}(k) \theta(k)-\phi^{T}(k) \hat{\theta}(k)\right) \\
= & \left(A_{\sigma(k)}+B_{\sigma(k)} K_{\sigma(k)}\right) x(k) \\
& +B_{\sigma(k)} V_{\sigma(k)} e_{\omega}(k)+B_{\sigma(k)} \phi^{T}(k) e_{\theta}(k) .
\end{aligned}
$$

$$
\begin{aligned}
& e_{\omega}(k+1)=(W+L V) e_{\omega}(k)+L \phi^{T}(k) e_{\theta}(k), \\
& e_{\theta}(k+1) \\
& =\left(\Gamma+L_{0} \phi^{T}(k)\right) e_{\theta}(k)+L_{0} B_{\sigma(k)} e_{\omega}(k) .
\end{aligned}
$$

\section{Main Results}

In this section, we are devoted to developing sufficient condition to solve the control problem formulated in the previous section.

Theorem 5. Consider system $\Sigma_{c}$. System $\Sigma_{c}$ is asymptotically stable if there exist matrices $K_{i}, L, L_{0}, P_{i}>0, S>0, T>$ $0, i \in \mathcal{N}$, such that the following inequalities hold:

$$
\begin{aligned}
& \Phi_{i}\left[\begin{array}{ccccccccc}
\Phi_{11 i} & 0 & 0 & \Phi_{15 i} & 0 & 0 & \Phi_{15 i} & 0 & 0 \\
* & -S & 0 & \Phi_{25 i} & \Phi_{26 i} & \Phi_{27 i} & 0 & \Phi_{26 i} & 0 \\
* & * & \Phi_{33} & 0 & 0 & \Phi_{37} & 0 & 0 & \Phi_{37} \\
* & * & * & -P_{l}^{-1} & 0 & 0 & 0 & 0 & 0 \\
* & * & * & * & -S^{-1} & 0 & 0 & 0 & 0 \\
* & * & * & * & * & -T^{-1} & 0 & 0 & 0 \\
* & * & * & * & * & * & -P_{l}^{-1} & 0 & 0 \\
* & * & * & * & * & * & * & -S^{-1} & 0 \\
* & * & * & * & * & * & * & * & -T^{-1}
\end{array}\right] \\
& <0, \\
& P_{i} \leq \lambda_{i} I, \\
& {\left[\begin{array}{cc}
-\delta_{2} I & L^{T} \\
L & -S^{-1}
\end{array}\right]<0,}
\end{aligned}
$$

where

$$
\begin{aligned}
\Phi_{11 i} & =-P_{i}, \\
\Phi_{15 i} & =\left(A_{i}+B_{i} K_{i}\right)^{T}, \\
\Phi_{26 i} & =(W+L V)^{T}, \\
\Phi_{33} & =-T+4 \lambda_{l} \phi^{2}\left\|B_{i}\right\|^{2}+3 \delta_{2} \phi^{2}+4 \delta_{3} \widehat{\phi}^{2}, \\
& \quad l=1,2, \ldots, N,
\end{aligned}
$$

$$
\begin{aligned}
& \Phi_{37}=\left(\Gamma+L_{0} E\right)^{T}, \\
& \Phi_{25 i}=\left(B_{i} V\right)^{T}, \\
& \Phi_{27 i}=\left(B_{i}^{T} L_{0}^{T}\right) .
\end{aligned}
$$

Proof. Choose a Lyapunov functional candidate as

$$
V(k)=V_{1}(k)+V_{2}(k)+V_{3}(k),
$$

where

$$
\begin{aligned}
& V_{1}(k)=x^{T}(k) P_{\sigma(k)} x(k), \\
& V_{2}(k)=e_{\omega}^{T}(k) S e_{\omega}(k), \\
& V_{3}(k)=e_{\theta}^{T}(k) T e_{\theta}(k) .
\end{aligned}
$$

Without loss of generality, we assume that $\sigma(k+1)=l$, $\sigma(k)=i, \forall i, l \in \mathcal{N}$. Then taking the forward difference $V_{1}(k)$ yields

$$
\begin{aligned}
\Delta V_{1}(k)= & V_{1}(k+1)-V_{1}(k) \\
= & x^{T}(k+1) P_{l} x(k+1)-x^{T}(k) P_{i} x(k) \\
= & \xi^{T}(k) \mathscr{A}_{i}^{T} P_{l} \mathscr{A}_{i} \xi(k)-x^{T}(k) P_{i} x(k) \\
= & x^{T}(k)\left(A_{i}+B_{i} K_{i}\right)^{T} P_{l}\left(A+B_{i} K_{i}\right) x(k) \\
& +e_{\omega}^{T}(k)\left(B_{i} V\right)^{T} P_{l}\left(B_{i} V\right) e_{\omega}(k) \\
& +e_{\theta}^{T}(k)\left(B_{i} \phi^{T}(k)\right)^{T} P_{l} B_{i} \phi^{T}(k) e_{\theta} \\
& +2 x^{T}(k)\left(A_{i}+B_{i} K_{i}\right)^{T} P_{l} B_{i} V e_{\omega} \\
& +2 x^{T}(k)\left(A_{i}+B_{i} K_{i}\right)^{T} P_{l} B_{i} \phi^{T}(k) e_{\theta} \\
& +2 e_{\omega}^{T}\left(B_{i} V\right)^{T} P_{l}\left(B_{i} \phi^{T}\right) e_{\theta}-x^{T}(k) P_{i} x(k),
\end{aligned}
$$


where $\xi^{T}(k)=\left[\begin{array}{lll}x^{T}(k) & e_{\omega}^{T}(k) & e_{\theta}^{T}(k)\end{array}\right] ; \mathscr{A}_{i}=\left[A_{i}+\right.$ $\left.B_{i} K_{i} \quad B_{i} V \quad B_{i} \phi^{T}(k)\right]$. Note that

$$
\begin{aligned}
& e_{\theta}^{T}(k)\left(B_{i} \phi^{T}(k)\right)^{T} P_{l} B_{i} \phi^{T}(k) e_{\theta} \\
& \leq\left\|e_{\theta}\right\|^{2}\left\|\phi^{T}(k)\right\|^{2}\left\|B_{i}\right\|^{2}\left\|P_{l}\right\| \leq \phi^{2}\left\|e_{\theta}\right\|^{2}\left\|B_{i}\right\|^{2}\left\|P_{l}\right\|, \\
& 2 x^{T}(k)\left(A_{i}+B_{i} K_{i}\right)^{T} P_{l} B_{i} \phi^{T}(k) e_{\theta} \\
& \leq x^{T}(k)\left(A_{i}+B_{i} K_{i}\right)^{T} P_{l}\left(A_{i}+B_{i} K_{i}\right) x(k) \\
& \quad+e_{\theta}^{T} \phi(k) B_{i}^{T} P_{l} B_{i} \phi^{T}(k) e_{\theta} \\
& \leq x^{T}(k)\left(A_{i}+B_{i} K_{i}\right)^{T} P_{l}\left(A_{i}+B_{i} K_{i}\right) x(k) \\
& \quad+\left\|e_{\theta}\right\|^{2}\|\phi(k)\|^{2}\left\|B_{i}\right\|^{2}\left\|P_{l}\right\| \\
& \leq x^{T}(k)\left(A_{i}+B_{i} K_{i}\right)^{T} P_{l}\left(A_{i}+B_{i} K_{i}\right) x(k) \\
& \quad+\phi^{2}\left\|e_{\theta}\right\|^{2}\left\|B_{i}\right\|^{2}\left\|P_{l}\right\|, \\
& 2 e_{\omega}^{T}\left(B_{i} V\right)^{T} P_{l}\left(B_{i} \phi^{T}\right) e_{\theta} \\
& \leq e_{\omega}^{T}\left(B_{i} V\right)^{T} P_{l} B_{i} V e_{\omega}+\phi^{2}\left\|e_{\theta}\right\|^{2}\left\|B_{i}\right\|^{2}\left\|P_{l}\right\| .
\end{aligned}
$$

Combining (18) and (19) yields

$$
\begin{aligned}
\Delta V_{1}(k) \leq & 2 x^{T}(k)\left(A_{i}+B_{i} K_{i}\right)^{T} P_{l}\left(A+B_{i} K_{i}\right) x(k) \\
& +2 e_{\omega}^{T}(k)\left(B_{i} V\right)^{T} P_{l}\left(B_{i} V\right) e_{\omega}(k) \\
& +2 x^{T}(k)\left(A_{i}+B_{i} K_{i}\right)^{T} P_{l} B_{i} V e_{\omega} \\
& +4 \lambda_{l} \phi^{2}\left\|e_{\theta}\right\|^{2}\left\|B_{i}\right\|^{2}-x^{T}(k) P_{i} x(k) .
\end{aligned}
$$

\section{Direct computation gives}

$$
\begin{aligned}
\Delta V_{2}(k)= & e_{\omega}(k+1) S e_{\omega}(k+1)-e_{\omega}^{T}(k) S \omega(k) \\
= & e_{\omega}^{T}(W+L V)^{T} S(W+L V) e_{\omega} \\
& +e_{\theta}^{T} \phi(k) L^{T} S L \phi^{T}(k) e_{\theta} \\
& +2 e_{\omega}^{T}(W+L V)^{T} S L \phi^{T}(k) e_{\theta} \\
& -e_{\omega}^{T}(k) S \omega(k) .
\end{aligned}
$$

Note that

$$
\begin{aligned}
& e_{\theta}^{T} \phi(k) L^{T} S L \phi^{T}(k) e_{\theta} \leq \delta_{2} \phi^{2}\left\|e_{\theta}\right\|^{2}, \\
& 2 e_{\omega}^{T}(W+L V)^{T} S L \phi^{T}(k) e_{\theta}
\end{aligned}
$$

$$
\begin{aligned}
\leq & e_{\omega}^{T}(W+L V)^{T} S(W+L V) e_{\omega} \\
& +e_{\theta}^{T} \phi(k) L^{T} S L \phi^{T}(k) e_{\theta} \\
\leq & e_{\omega}^{T}(W+L V)^{T} S(W+L V) e_{\omega}+\delta_{2} \phi^{2}\left\|e_{\theta}\right\|^{2} .
\end{aligned}
$$

By substituting (22) into (21), we have

$$
\begin{aligned}
\Delta V_{2}(k) \leq & 2 e_{\omega}^{T}(W+L V)^{T} S(W+L V) e_{\omega} \\
& +2 e_{\theta}^{T} \phi(k) L^{T} S L \phi^{T}(k) e_{\theta}+3 \delta_{2} \phi^{2}\left\|e_{\theta}\right\|^{2} \\
& -e_{\omega}^{T}(k) S \omega(k) .
\end{aligned}
$$

Computing the difference $V_{3}(k)$ yields

$$
\begin{aligned}
\Delta V_{3}(k)= & e_{\theta}^{T}(k+1) T e_{\theta}(k+1)-e_{\theta}^{T}(k) T e_{\theta}(k) \\
= & e_{\theta}^{T}\left(\Gamma+L_{0} \phi^{T}(k)\right)^{T} Q\left(\Gamma+L_{0} \phi^{T}(k)\right) e_{\theta} \\
& +2 e_{\theta}^{T}\left(\Gamma+L_{0} \phi^{T}(k)\right)^{T} Q L_{0} B_{i} e_{\omega}(k) \\
& +e_{\omega}^{T} B_{i}^{T} L_{0}^{T} Q L_{0} B_{i} e_{\omega}-e_{\theta}^{T}(k) T e_{\theta}(k) .
\end{aligned}
$$

Note that

$$
e_{\theta}^{T}\left(\Gamma+L_{0} \phi^{T}(k)\right)^{T} Q\left(\Gamma+L_{0} \phi^{T}(k)\right) e_{\theta}
$$

$$
\begin{aligned}
& =e_{\theta}^{T}\left(\Gamma+L_{0}\left(E+\widehat{\phi}^{T}(k)\right)\right)^{T} \\
& \cdot Q\left(\Gamma+L_{0}\left(E+\widehat{\phi}^{T}(k)\right)\right) e_{\theta}=e_{\theta}^{T}\left(\Gamma+L_{0} E\right)^{T}
\end{aligned}
$$$$
\cdot Q\left(\Gamma+L_{0} E\right) e_{\theta}+2 e_{\theta}^{T}\left(\Gamma+L_{0} E\right)^{T} Q L_{0} \widehat{\phi}^{T}(k) e_{\theta}
$$$$
+e_{\theta}^{T} \widehat{\phi}(k) L_{0}^{T} Q L_{0} \widehat{\phi}^{T}(k) e_{\theta},
$$

$$
\begin{aligned}
& 2 e_{\theta}^{T}\left(\Gamma+L_{0} E\right)^{T} Q L_{0} \widehat{\phi}^{T}(k) e_{\theta} \leq e_{\theta}^{T}\left(\Gamma+L_{0} E\right)^{T} \\
& \cdot Q\left(\Gamma+L_{0} E\right) e_{\theta}+e_{\theta}^{T} \widehat{\phi}(k) L_{0}^{T} Q L_{0} \widehat{\phi}^{T}(k) e_{\theta}, \\
& 2 e_{\theta}^{T}\left(\Gamma+L_{0} \phi^{T}(k)\right)^{T} Q L_{0} B_{i} e_{\omega}(k) \\
& =2 e_{\theta}^{T}\left(\Gamma+L_{0} E+L_{0} \widehat{\phi}^{T}(k)\right)^{T} Q L_{0} B_{i} e_{\omega}(k) \\
& =2 e_{\theta}^{T}\left(\Gamma+L_{0} E\right)^{T} Q L_{0} B_{i} e_{\omega}(k)+2 e_{\theta}^{T}\left(L_{0} \widehat{\phi}^{T}(k)\right)^{T} \\
& \cdot Q L_{0} B_{i} e_{\omega}(k) \leq 2 e_{\theta}^{T}\left(\Gamma+L_{0} E\right)^{T} Q L_{0} B_{i} e_{\omega}(k) \\
& +e_{\theta}^{T} \widehat{\phi} L_{0}^{T} Q L_{0} \widehat{\phi}^{T} e_{\theta}+e_{\omega}^{T} B_{i}^{T} L_{0} Q L_{0}^{T} B_{i} e_{\omega} .
\end{aligned}
$$


By combining (24) and (25), we have

$$
\begin{aligned}
\Delta V_{3}(k) \leq & 2 e_{\theta}^{T}\left(\Gamma+L_{0} E\right)^{T} \mathrm{Q}\left(\Gamma+L_{0} E\right) e_{\theta} \\
& +2 e_{\theta}^{T}\left(\Gamma+L_{0} E\right)^{T} \mathrm{Q} L_{0} B_{i} e_{\omega}(k) \\
& +2 e_{\omega}^{T} B_{i}^{T} L_{0} Q L_{0}^{T} B_{i} e_{\omega}+4 e_{\theta}^{T} \widehat{\phi} L_{0}^{T} Q L_{0} \widehat{\phi}^{T} e_{\theta} \\
& -e_{\theta}^{T}(k) T e_{\theta}(k) \\
\leq & 2 e_{\theta}^{T}\left(\Gamma+L_{0} E\right)^{T} \mathrm{Q}\left(\Gamma+L_{0} E\right) e_{\theta} \\
& +2 e_{\theta}^{T}\left(\Gamma+L_{0} E\right)^{T} Q L_{0} B_{i} e_{\omega}(k) \\
& +2 e_{\omega}^{T} B_{i}^{T} L_{0} Q L_{0}^{T} B_{i} e_{\omega}+4 \delta_{3}\|\hat{\phi}\|^{2}\left\|e_{\theta}\right\|^{2} \\
& -e_{\theta}^{T}(k) T e_{\theta}(k) .
\end{aligned}
$$

From (18)-(26), and by some manipulations, we obtain

$$
\Delta V(k) \leq 2 x^{T}(k)\left(A_{i}+B_{i} K_{i}\right)^{T} P_{l}\left(A+B_{i} K_{i}\right) x(k)
$$

$$
\begin{aligned}
& +2 e_{\omega}^{T}(k)\left(B_{i} V\right)^{T} P_{l}\left(B_{i} V\right) e_{\omega}(k) \\
& +2 x^{T}(k)\left(A_{i}+B_{i} K_{i}\right)^{T} P_{l} B_{i} V e_{\omega} \\
& +4 \lambda_{l} \phi^{2}\left\|e_{\theta}\right\|^{2}\left\|B_{i}\right\|^{2}-x^{T}(k) P_{i} x(k) \\
& +2 e_{\omega}^{T}(W+L V)^{T} S(W+L V) e_{\omega} \\
& +3 \delta_{2} \phi^{2}\left\|e_{\theta}\right\|^{2}-e_{\omega}^{T}(k) S \omega(k) \\
& +2 e_{\theta}^{T}\left(\Gamma+L_{0} E\right)^{T} Q\left(\Gamma+L_{0} E\right) e_{\theta} \\
& +2 e_{\theta}^{T}\left(\Gamma+L_{0} E\right)^{T} Q L_{0} B_{i} e_{\omega}(k) \\
& +2 e_{\omega}^{T} B_{i}^{T} L_{0} Q L_{0}^{T} B_{i} e_{\omega}+4 \delta_{3}\|\hat{\phi}\|^{2}\left\|e_{\theta}\right\|^{2} \\
& -e_{\theta}^{T}(k) T e_{\theta}(k)=\xi^{T}(k) \Phi_{1 i} \xi(k),
\end{aligned}
$$

where

$$
\begin{aligned}
\Phi_{1 i} & =\left[\begin{array}{ccc}
\Phi_{11 i} & \Phi_{12 i} & 0 \\
* & \Phi_{22 i} & \Phi_{23 i} \\
* & * & \Phi_{33 i}
\end{array}\right], \\
\Phi_{11 i} & =2\left(A_{i}+B_{i} K_{i}\right)^{T} P_{l}\left(A+B_{i} K_{i}\right)-P_{i}, \\
\Phi_{12 i} & =\left(A_{i} B_{i} K_{i}\right)^{T} P_{l} B_{i} V,
\end{aligned}
$$

$$
\begin{aligned}
\Phi_{22 i}= & 2(W+L V)^{T} S(W+L V)+\left(B_{i} V\right)^{T} P_{l}\left(B_{i} V\right) \\
& +B_{i}^{T} L_{0}^{T} Q L_{0} B_{i}, \\
\Phi_{23 i}= & B_{i}^{T} L_{0}^{T} Q\left(\Gamma+L_{0} E\right), \\
\Phi_{33 i}= & 2\left(\Gamma+L_{0} E\right)^{T} Q\left(\Gamma+L_{0} E\right)-Q+4 \lambda_{l} \phi^{2}\left\|B_{i}\right\|^{2} \\
& +3 \delta_{2} \phi^{2}+4 \delta_{3} \widehat{\phi}^{2} .
\end{aligned}
$$

By applying Schur complement formula, we obtain $\Delta V(k)<0$ if (11) is true. Therefore, it is easy to see that the closed-loop system $\Sigma_{c}$ is asymptotically stable by the Lyapunov function stability theorem. This completes the proof.

Remark 6. In Theorem 5, a sufficient condition is derived to guarantee system's asymptotical stability, but the condition is presented by some nonlinear matrix inequalities. In order to solve the controller and observer gains, we cast the inequalities (11)-(14) into linear matrix inequalities.

By premultiplying and postmultiplying $\operatorname{diag}\left\{P_{i}^{-1}, I, I, I\right.$, $S, T, I, S, T\}=\operatorname{diag}\left\{X_{i}, I, I, I, S, T, I, S, T\right\}$ with (11), premultiplying and postmultiplying $\operatorname{diag}\{I, S\}$ with (13), and premultiplying and postmultiplying $\operatorname{diag}\{I, T\}$ with (14), we have

$\bar{\Phi}_{i}$

$$
\begin{aligned}
& {\left[\begin{array}{ccccccccc}
\bar{\Phi}_{11 i} & 0 & 0 & \bar{\Phi}_{15 i} & 0 & 0 & \bar{\Phi}_{15 i} & 0 & 0 \\
* & -S & 0 & \Phi_{25 i} & \bar{\Phi}_{26 i} & \bar{\Phi}_{27 i} & 0 & \bar{\Phi}_{26 i} & 0 \\
* & * & \Phi_{33} & 0 & 0 & \Phi_{37} & 0 & 0 & \Phi_{37} \\
* & * & * & -X_{l} & 0 & 0 & 0 & 0 & 0 \\
* & * & * & * & -S & 0 & 0 & 0 & 0 \\
* & * & * & * & * & -T & 0 & 0 & 0 \\
* & * & * & * & * & * & -X_{l} & 0 & 0 \\
* & * & * & * & * & * & * & -S & 0 \\
* & * & * & * & * & * & * & * & -T
\end{array}\right]} \\
& <0,
\end{aligned}
$$

$$
\begin{aligned}
& X_{i} \geq \frac{1}{\lambda_{i}} I, \\
& {\left[\begin{array}{cc}
-\delta_{2} I & \bar{L}^{T} \\
\bar{L} & -S
\end{array}\right]<0,} \\
& {\left[\begin{array}{cc}
-\delta_{3} I & \bar{L}_{0}^{T} \\
\bar{L}_{0} & -T
\end{array}\right]<0,}
\end{aligned}
$$


where

$$
\begin{aligned}
& \Phi_{11 i}=-X_{i}, \\
& \Phi_{15 i}=\left(A_{i} X_{i}+B_{i} \bar{K}_{i}\right)^{T}, \\
& \Phi_{26 i}=(S W+\bar{L} V)^{T}, \\
& \Phi_{33}=-T+4 \lambda_{l} \phi^{2}\left\|B_{i}\right\|^{2}+3 \delta_{2} \phi^{2}+4 \delta_{3} \hat{\phi}^{2}, \\
& \qquad \quad l=1,2, \ldots, N, \\
& \Phi_{37}=\left(T \Gamma+\bar{L}_{0} E\right)^{T}, \\
& \Phi_{25 i}=\left(B_{i} V\right)^{T}, \\
& \Phi_{27 i}=\left(B_{i}^{T} \bar{L}_{0}^{T}\right),
\end{aligned}
$$

with $K_{i}=\bar{K}_{i} X_{i}^{-1}, L=S^{-1} \bar{L}$, and $L_{0}=T^{-1} \bar{L}_{0}$.

Remark 7. Although we first design a disturbance observer and adaptive control law to estimate the disturbance and unknown parameter, respectively, and then the composite controller is constructed based on the estimation values and feedback control law, the disturbance observer and the composite controller are solved simultaneously in Theorem 5. By solving conditions (29), we can get the values of controller and observer gains $K_{i}, L_{0}$, and $L$.

Now, we consider the case: switched system with Assumptions 1 and 2 has one subsystem $(N=1)$ :

$$
x(k+1)=A x(k)+B\left(u(k)+d_{1}(k)+\phi^{T}(k) \theta(k)\right),
$$

with

$$
\begin{aligned}
\omega(k+1) & =W \omega(k), \\
d_{1}(k) & =V \omega(k), \\
\theta(k+1) & =\Gamma \theta(k) .
\end{aligned}
$$

And the composite adaptive antidisturbance controller is designed:

$$
\begin{aligned}
& u(k)=K x(k)-\widehat{d}_{1}(k)-\phi^{T}(k) \hat{\theta}(k), \\
& \widehat{\omega}(k)=v(k)-L x(k), \\
& v(k+1)=W \widehat{\omega}(k)+L\left(A_{\sigma(k)} x(k)\right. \\
& \left.\quad+B_{\sigma(k)}\left(u(k)+\widehat{d}_{1}(k)+\phi^{T}(k) \widehat{\theta}(k)\right)\right), \\
& \widehat{d}_{1}(k)=V \widehat{\omega}(k), \\
& \widehat{\theta}(k)=v_{0}(k)-L_{0} x(k), \\
& v_{0}(k+1)=\Gamma \widehat{\theta}(k)+L_{0}\left(A_{\sigma(k)} x(k)\right. \\
& \left.\quad+B_{\sigma(k)}\left(u(k)+\widehat{d}_{1}(k)+\phi^{T}(k) \widehat{\theta}(k)\right)\right),
\end{aligned}
$$

where $K, L$, and $L_{0}$ are controller and observer gains to be determined later.

For such a case, the closed-loop system becomes a class of discrete-time nonlinear system effectively operating at one of the subsystems all the time, and it can be described by

$$
\begin{aligned}
x(k+1)= & (A+B K) x(k)+B V e_{\omega}(k) \\
& +B \phi^{T}(k) e_{\theta}(k), \\
e_{\omega}(k+1)= & (W+L V) e_{\omega}(k)+L \phi^{T}(k) e_{\theta}(k), \\
e_{\theta}(k+1)= & \left(\Gamma+L_{0} \phi^{T}(k)\right) e_{\theta}(k)+L_{0} B_{\sigma(k)} e_{\omega}(k) .
\end{aligned}
$$

Corollary 8. Consider system (35). System (35) is asymptotically stable if there exist matrices $K, L, L_{0}, P>0, S>0$, and $T>0$ such that the following inequalities hold:

$\Phi$

$$
=\left[\begin{array}{ccccccccc}
\Phi_{11} & 0 & 0 & \Phi_{15} & 0 & 0 & \Phi_{15} & 0 & 0 \\
* & -S & 0 & \Phi_{25} & \Phi_{26} & \Phi_{27} & 0 & \Phi_{26} & 0 \\
* & * & \Phi_{33} & 0 & 0 & \Phi_{37} & 0 & 0 & \Phi_{37} \\
* & * & * & -P^{-1} & 0 & 0 & 0 & 0 & 0 \\
* & * & * & * & -S^{-1} & 0 & 0 & 0 & 0 \\
* & * & * & * & * & -T^{-1} & 0 & 0 & 0 \\
* & * & * & * & * & * & -P^{-1} & 0 & 0 \\
* & * & * & * & * & * & * & -S^{-1} & 0 \\
* & * & * & * & * & * & * & * & -T^{-1}
\end{array}\right]
$$

$<0$,

$P \leq \lambda I$,

$$
\begin{aligned}
& {\left[\begin{array}{cc}
-\delta_{2} I & L^{T} \\
L & -S^{-1}
\end{array}\right]<0,} \\
& {\left[\begin{array}{cc}
-\delta_{3} I & L_{0}^{T} \\
L_{0} & -T^{-1}
\end{array}\right]<0,}
\end{aligned}
$$

where

$$
\begin{aligned}
& \Phi_{11}=-P, \\
& \Phi_{15}=(A+B K)^{T}, \\
& \Phi_{26}=(W+L V)^{T}, \\
& \Phi_{33}=-T+4 \lambda_{l} \phi^{2}\|B\|^{2}+3 \delta_{2} \phi^{2}+4 \delta_{3} \widehat{\phi}^{2} \\
& \Phi_{37}=\left(\Gamma+L_{0} E\right)^{T}, \\
& \Phi_{25}=(B V)^{T}, \\
& \Phi_{27}=\left(B^{T} L_{0}^{T}\right) .
\end{aligned}
$$

Remark 9. To the best of the authors' knowledge, this is the first time that the disturbance observer based on adaptive control strategy is applied to the control problem for discrete time with multiple disturbances. 


\section{A Numerical Example}

Now, we provide an example to show the effectiveness of the main result in this paper.

Consider discrete-time switched system with parameters as follows:

$$
\begin{aligned}
& A_{1}=\left[\begin{array}{cc}
-0.3 & -0.1 \\
0.5 & -0.1
\end{array}\right], \\
& A_{2}=\left[\begin{array}{cc}
-0.1 & 0.2 \\
0 & -0.5
\end{array}\right], \\
& B_{1}=\left[\begin{array}{c}
0.01 \\
-0.2
\end{array}\right], \\
& B_{2}=\left[\begin{array}{c}
-0.02 \\
0.2
\end{array}\right] .
\end{aligned}
$$

The disturbance model is presented by the following parameters:

$$
\begin{aligned}
W & =\left[\begin{array}{cc}
0.8776 & 0.4794 \\
-0.4794 & 0.8776
\end{array}\right], \\
V & =\left[\begin{array}{ll}
10 & 0
\end{array}\right] .
\end{aligned}
$$

The parameter of model (33) is given as

$$
\Gamma=\left[\begin{array}{cc}
0.7071 & -0.7071 \\
0.7071 & 0.7071
\end{array}\right] \text {. }
$$
lows:

The controller gains and observer gain are listed as fol-

$$
\begin{aligned}
K_{1} & =\left[\begin{array}{ll}
2.5738 & -0.4718
\end{array}\right], \\
K_{2} & =\left[\begin{array}{ll}
2.5777 & -0.5513
\end{array}\right], \\
L & =\left[\begin{array}{cc}
0.0708 & -0.3454 \\
-0.0361 & 0.2504
\end{array}\right], \\
L_{0} & =\left[\begin{array}{ll}
0.0612 & 0.0235 \\
0.0155 & -0.0189
\end{array}\right] .
\end{aligned}
$$

The initial value of the states is chosen as $\phi(s)=$ $\left[\begin{array}{ll}-2 & 0.5\end{array}\right]^{T}$. In order to illustrate the effectiveness of the proposed method, we consider two kinds of switching signal: determinate switching signal and stochastic switching signal.

(i) Determinate Switching Signal. Suppose the switching sequence as $121212 \cdots$. In Figure 1, system states are shown, in which we can see the proposed method can achieve a good disturbance rejection performance in spite of external disturbance and unknown parameters. Figure 2 depicts the curves of control input. In order to demonstrate the effectiveness of the proposed observer, curves of disturbances and disturbance estimation are presented in Figure 3 and curves of unknown parameters and parameters estimation

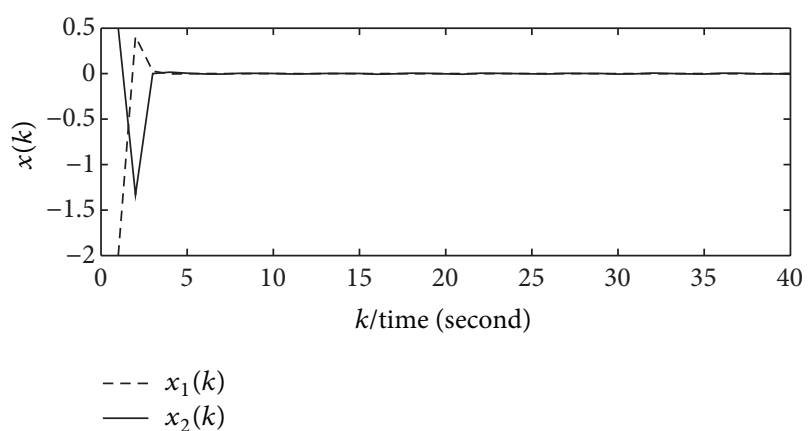

Figure 1: Response curves of system states $x(k)$.

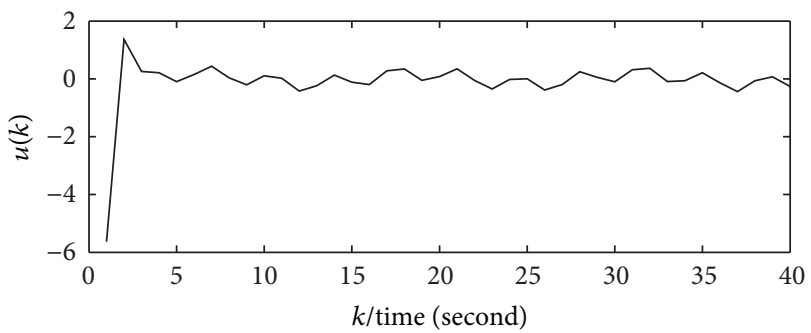

FIGURE 2: Curves of system input.

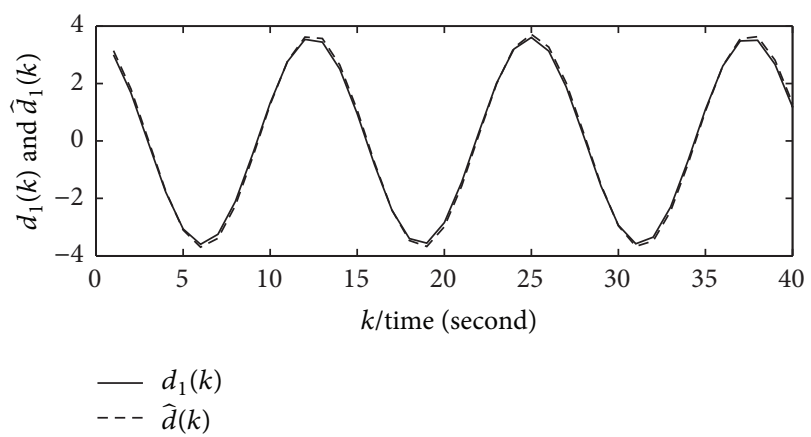

FIGURE 3: Curves of disturbances and disturbances estimation.

are described in Figure 4. From these figures, we can see the disturbance observer and adaptive control law can effectively estimate external disturbance and unknown parameters, respectively. According to the simulation results, we can conclude that the proposed scheme can guarantee system has a satisfactory performance in presence of external disturbances and unknown parameters.

(ii) Stochastic Switching Signal. The simulation results are presented as follows. From Figure 5, we can see the proposed method can obtain a good performance in spite of stochastic switching signal. The switching signal is presented in Figure 6.

Remark 10. In this paper, the problem of composite adaptive antidisturbance control is considered for a class of switched systems with arbitrary switching signal; namely, if we design different switching signal, the system can be stable under the switching signal. The simulation results demonstrate this point. 

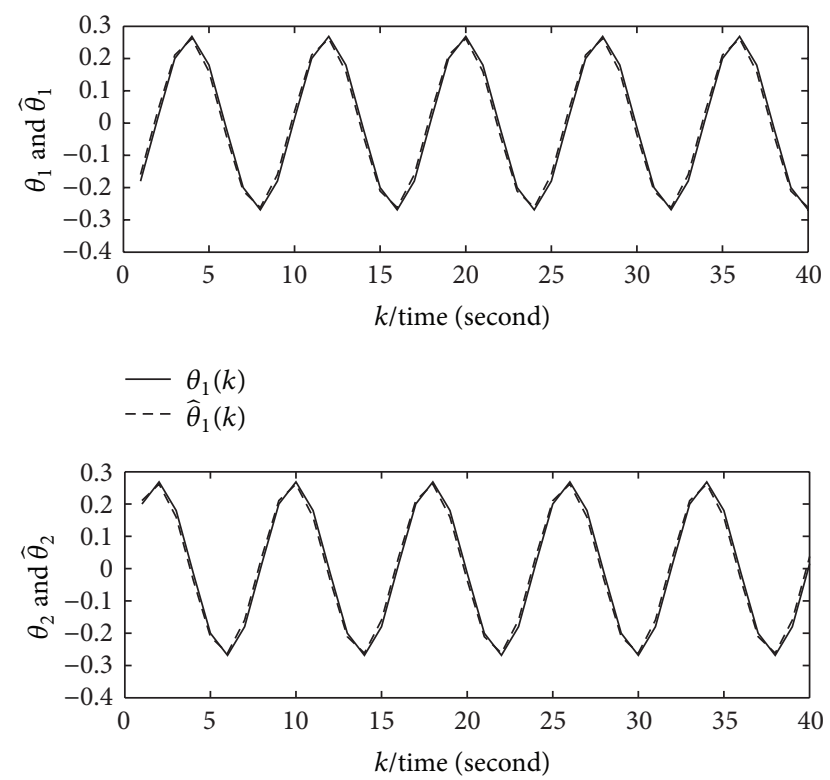

$-\theta_{2}(k)$

FIGURE 4: Curves of unknown parameters and parameters estimation.

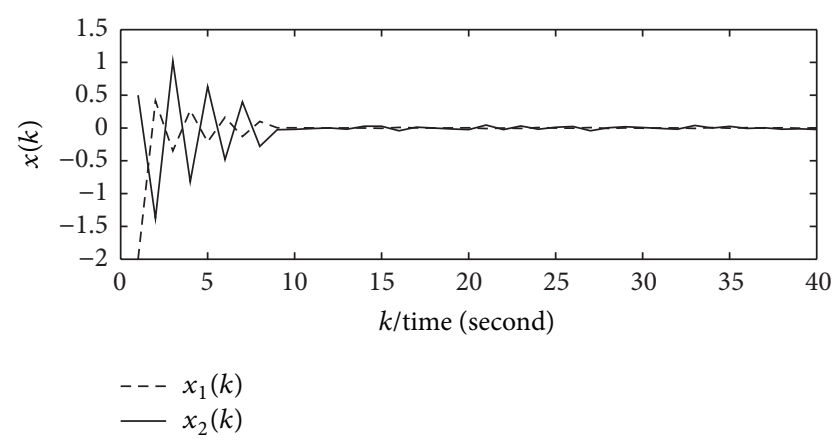

FIGURE 5: Response curves of system states $x(k)$ with stochastic switching signal.

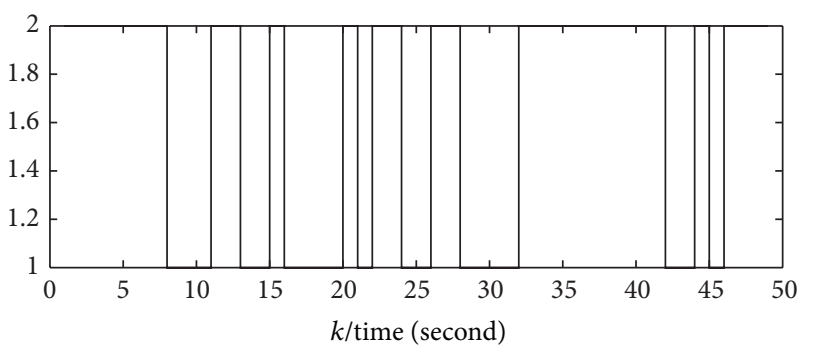

— Switching signal

FIGURE 6: Curves of stochastic switching signal.

\section{Conclusions}

The problem of adaptive antidisturbance control for discretetime switched systems subject to disturbances has been investigated. The composite adaptive observers have been constructed to estimate disturbance and unknown parameters. A composite antidisturbance adaptive controller has been given based on the output of observer and conventional feedback control law. Some sufficient conditions have been developed via linear matrix inequalities technique. A numerical example has been provided to show the effectiveness of the proposed algorithm.

\section{Competing Interests}

The authors declare that they have no competing interests.

\section{Acknowledgments}

This work was supported in part by the National Natural Science Foundation of China (nos. 61403227, 61304059, 61304153, and 61273123), in part by National Science Foundation of Shandong Province under Grant no. ZR2013FQ016, and in part by Project supported by the Zhejiang Open Foundation of the Most Important Subjects, Research Plan for Application Base and Advanced Technology of Tianjin (no. 15JCQNJC04200), and the High School Science and Technology Development Fund Project of Tianjin (no. 20120828).

\section{References}

[1] J. C. Doyle, K. Glover, P. P. Khargonekar, and B. A. Francis, "State-space solutions to standard $H_{2}$ and $H_{\infty}$ control problems," IEEE Transactions on Automatic Control, vol. 34, no. 8, pp. 831-847, 1989.

[2] X.-H. Chang, J. H. Park, and J. P. Zhou, "Robust static output feedback $H_{\infty}$ control design for linear systems with polytopic uncertainties," Systems and Control Letters, vol. 85, pp. 23-32, 2015.

[3] Z. Ding, "Global stabilization and disturbance suppression of a class of nonlinear systems with uncertain internal model," Automatica, vol. 39, no. 3, pp. 471-479, 2003.

[4] Z. Ding, "Universal disturbance rejection for nonlinear systems in output feedback form," IEEE Transactions on Automatic Control, vol. 48, no. 7, pp. 1222-1226, 2003.

[5] Y. Feng, X. Yu, and Z. Man, "Non-singular terminal sliding mode control of rigid manipulators," Automatica, vol. 38, no. 12, pp. 2159-2167, 2002.

[6] J. Yang, S. Li, J. Su, and X. Yu, "Continuous nonsingular terminal sliding mode control for systems with mismatched disturbances," Automatica, vol. 49, no. 7, pp. 2287-2291, 2013.

[7] L. Guo and M. Tomizuka, "High-speed and high-precision motion control with an optimal hybrid feedforward controller," IEEE/ASME Transactions on Mechatronics, vol. 2, no. 2, pp. 110$122,1997$.

[8] Y. H. Huang and W. Messner, "A novel disturbance observer design for magnetic hard drive servo system with a rotary actuator," IEEE Transactions on Magnetics, vol. 34, no. 4, pp. 1892-1894, 1998.

[9] W.-H. Chen, D. J. Ballance, P. J. Gawthrop, and J. O’Reilly, “A nonlinear disturbance observer for robotic manipulators," IEEE Transactions on Industrial Electronics, vol. 47, no. 4, pp. 932-938, 2000 . 
[10] X. S. Chen, J. Yang, S. H. Li, and Q. Li, "Disturbance observer based multi-variable control of ball mill grinding circuits," Journal of Process Control, vol. 19, no. 7, pp. 1205-1213, 2009.

[11] J. Yang, S. Li, X. Chen, and Q. Li, "Disturbance rejection of ball mill grinding circuits using DOB and MPC," Powder Technology, vol. 198, no. 2, pp. 219-228, 2010.

[12] S. H. Li, H. B. Sun, and C. Y. Sun, "Composite controller design for an airbreathing hypersonic vehicle," Proceedings of the Institution of Mechanical Engineers Part I: Journal of Systems and Control Engineering, vol. 226, no. 5, pp. 651-664, 2012.

[13] H. B. Sun, S. H. Li, and C. Y. Sun, "Finite time integral sliding mode control of hypersonic vehicles," Nonlinear Dynamics, vol. 73, no. 1-2, pp. 229-244, 2013.

[14] H. B. Sun, S. H. Li, and S. M. Fei, "A composite control scheme for $6 \mathrm{DOF}$ spacecraft formation control," Acta Astronautica, vol. 69, no. 7-8, pp. 595-611, 2011.

[15] H. B. Sun and S. H. Li, "Composite control method for stabilizing spacecraft attitude in terms of Rodrigues parameters," Chinese Journal of Aeronautics, vol. 26, no. 3, pp. 687-696, 2013.

[16] W.-H. Chen, "Disturbance observer based control for nonlinear systems," IEEE/ASME Transactions on Mechatronics, vol. 9, no. 4, pp. 706-710, 2004.

[17] L. Guo and W. H. Chen, "Disturbance attenuation and rejection for a class of nonlinear systems via DOBC approach," International Journal of Robust and Nonlinear Control, vol. 15, no. 3, pp. 109-125, 2005.

[18] S. H. Li, J. Yang, W. H. Chen, and X. S. Chen, Disturbance Observer Based Control: Methods and Application, CRC Press, 2014.

[19] L. Guo and S. Y. Cao, Anti-Disturbance Control for Systems with Multiple Disturbances, CRC Press, 2013.

[20] X. Wei and L. Guo, "Composite disturbance-observer-based control and $H_{\infty}$ control for complex continuous models," International Journal of Robust and Nonlinear Control, vol. 20, no. 1, pp. 106-118, 2010.

[21] Y. K. Li, H. B. Sun, G. D. Zong, and L. L. Hou, "Disturbanceobserver-based-control and $L_{2}-L_{\infty}$ resilient control for Markovian jump non-linear systems with multiple disturbances and its application to single robot arm system," IET Control Theory \& Applications, vol. 10, no. 2, pp. 226-233, 2016.

[22] X. J. Wei and L. Guo, "Composite disturbance-observer-based control and terminal sliding mode control for non-linear systems with disturbances," International Journal of Control, vol. 82, no. 6, pp. 1082-1098, 2009.

[23] X. Liu, Z. Liu, J. Shan, and H. Sun, "Anti-disturbance autopilot design for missile system via finite time integral sliding mode control method and nonlinear disturbance observer technique," Transactions of the Institute of Measurement and Control, 2015.

[24] L. Guo and X.-Y. Wen, "Hierarchical anti-disturbance adaptive control for non-linear systems with composite disturbances and applications to missile systems," Transactions of the Institute of Measurement and Control, vol. 33, no. 8, pp. 942-956, 2011.

[25] H. B. Sun and L. Guo, "Composite adaptive disturbance observer based control and back-stepping method for nonlinear system with multiple mismatched disturbances," Journal of the Franklin Institute, vol. 351, no. 2, pp. 1027-1041, 2014.

[26] H. Sun and L. Guo, "Neural network based DOBC for a class of nonlinear systems with unmatched disturbances," IEEE Transactions on Neural Networks and Learning Systems, 2016.

[27] B. Xu, Y. P. Pan, D. W. Wang, and F. C. Sun, "Discrete-time hypersonic flight control based on extreme learning machine," Neurocomputing, vol. 128, pp. 232-241, 2014.
[28] B. Xu and Y. Zhang, "Neural discrete back-stepping control of hypersonic flight vehicle with equivalent prediction model," Neurocomputing, vol. 154, pp. 337-346, 2015.

[29] X. J. Wei, N. Chen, C. H. Deng, X. H. Liu, and M. Q. Tang, "Composite stratified anti disturbance control for a class of MIMO discrete-time systems with nonlinearity," International Journal of Robust and Nonlinear Control, vol. 22, no. 4, pp. 453472, 2012.

[30] H. B. Sun and L. L. Hou, "Composite anti-disturbance control for a discrete-time time-varying delay system with actuator failures based on a switching method and a disturbance observer," Nonlinear Analysis: Hybrid Systems, vol. 14, pp. 126-138, 2014.

[31] D. Liberzon and R. Tempo, "Common Lyapunov functions and gradient algorithms," IEEE Transactions on Automatic Control, vol. 49, no. 6, pp. 990-994, 2004.

[32] N. H. El-Farra, P. Mhaskar, and P. D. Christofides, "Output feedback control of switched nonlinear systems using multiple Lyapunov functions," Systems and Control Letters, vol. 54, no. 12, pp. 1163-1182, 2005.

[33] L. L. Hou, G. D. Zong, Y. Q. Wu, and Y. C. Cao, "Exponential $l_{2}-l_{\infty}$ output tracking control for discrete-time switched system with time-varying delay," International Journal of Robust and Nonlinear Control, vol. 22, pp. 1175-1194, 2012.

[34] H. Sun, G. Zong, and L. Hou, " $H_{\infty}$ guaranteed cost filtering for uncertain discrete-time switched systems with multiple timevarying delays," Journal of Dynamic Systems, Measurement, and Control, vol. 133, no. 1, Article ID 014503, 4 pages, 2010.

[35] H.-B. Sun and L.-L. Hou, "Composite disturbance observerbased control and $H_{\infty}$ output tracking control for discretetime switched systems with time-varying delay," Mathematical Problems in Engineering, vol. 2013, Article ID 698935, 12 pages, 2013.

[36] J. Daafouz, P. Riedinger, and C. Iung, "Stability analysis and control synthesis for switched systems: a switched Lyapunov function approach," IEEE Transactions on Automatic Control, vol. 47, no. 11, pp. 1883-1887, 2002. 


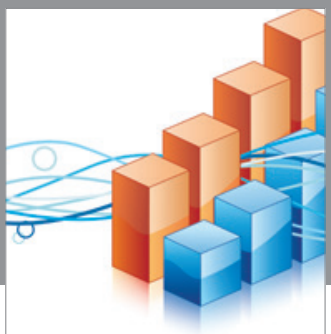

Advances in

Operations Research

vatem alat4

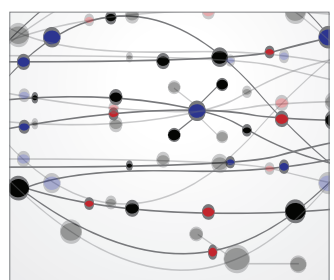

\section{The Scientific} World Journal
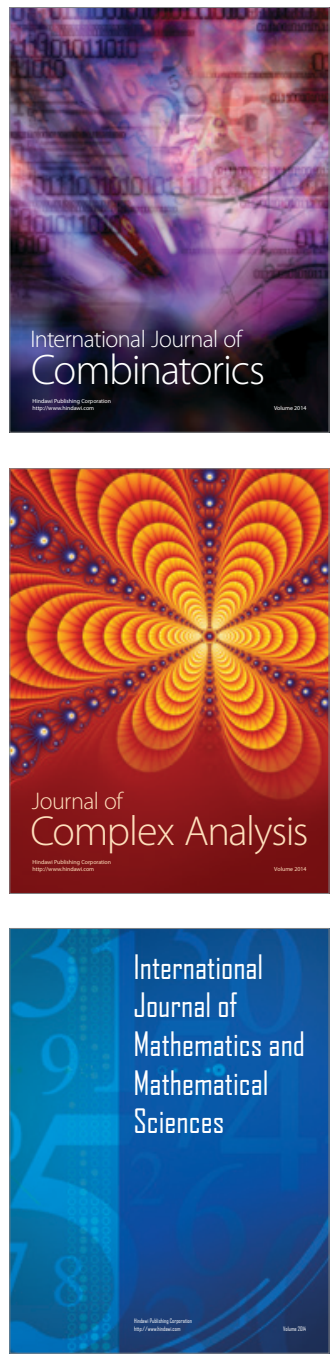
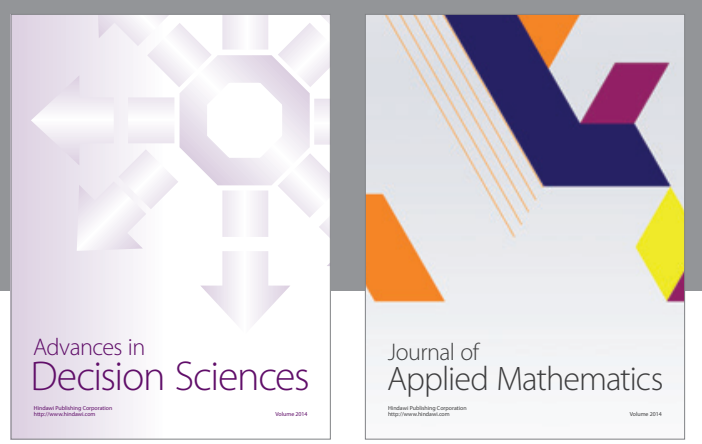

Algebra

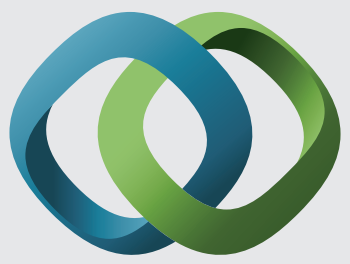

\section{Hindawi}

Submit your manuscripts at

http://www.hindawi.com
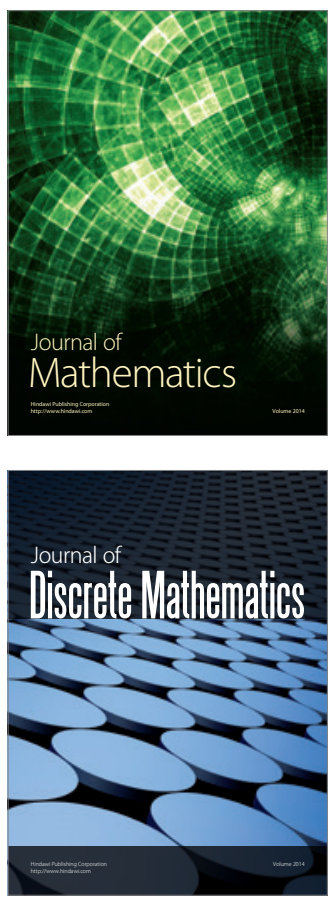

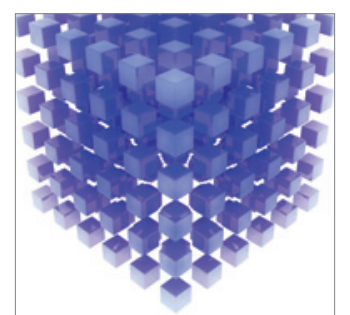

Mathematical Problems in Engineering
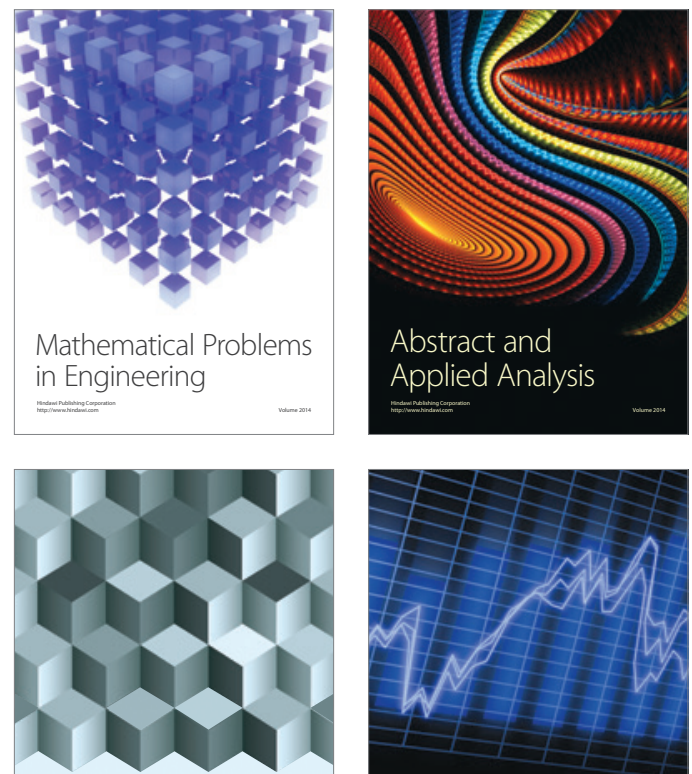

Journal of

Function Spaces

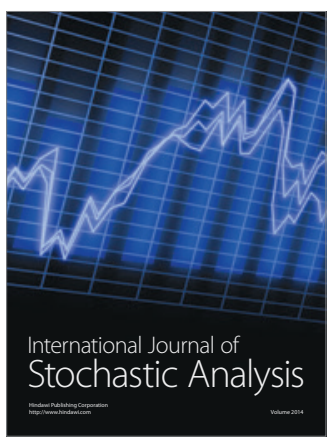

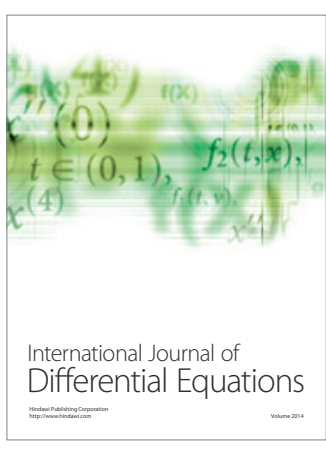
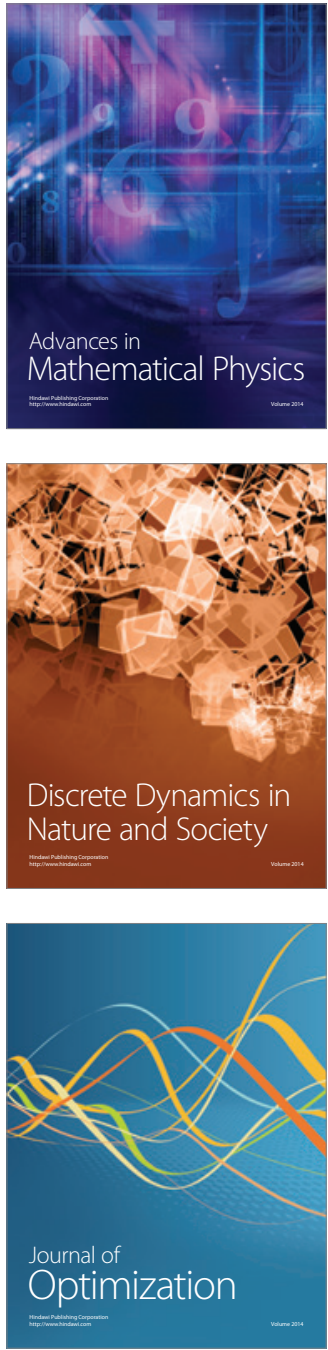